\title{
Neoantigen-based Melanoma-Poly-ICLC Vaccine
}

National Cancer Institute

\section{Source}

National Cancer Institute. Neoantigen-based Melanoma-Poly-ICLC Vaccine. NCI

Thesaurus. Code C112003.

A peptide-based melanoma cancer vaccine consisting of neoantigens and peptides derived from patient-specific melanoma immunogenic epitopes, combined with the immunostimulant poly-ICLC with potential immunomodulating and antineoplastic activities. Vaccination with the neoantigen-based melanoma vaccine stimulates the host immune system to mount a cytotoxic $T$ lymphocyte $(C T L)$ response against tumor cells expressing the neoantigens, which results in tumor cell lysis. The adjuvant poly-ICLC, composed of double-stranded RNA molecules of polyinosinic-polycytidylic acid stabilized with poly L-lysine in carboxymethylcellulose, is a lig and for toll-like receptor-3 (TLR3) and induces the release of cytokines which may help to boost the immune response against the selected neoantigens. 